\title{
Photon self-energy in a color superconductor
}

\author{
Daniel F. Litim* and Cristina Manuel ${ }^{\dagger}$ \\ Theory Division, CERN, CH-1211 Geneva 23, Switzerland \\ (Received 30 May 2001; published 5 October 2001)
}

\begin{abstract}
In a color superconductor the diquark condensates break spontaneously both the color and ordinary electromagnetism, leaving a remaining rotated $U(1)$ symmetry unbroken. The gauge interactions associated with this rotated symmetry may be considered as the in-medium electromagnetism. We compute the in-medium photon self-energy in the presence of diquark condensates at high baryonic density and weak coupling. This is done to one-loop order for the cases of two and three quark flavors. For vanishing temperature, a detailed discussion is given of the low momentum behavior of the photon polarization tensor. A simple physical picture for the propagation of light in color superconducting media is obtained. The main new effect is due to the diquark condensates, which lead to a strong dielectric constant of the medium. The magnetic permeability remains unchanged, because the primary condensates have vanishing spin and angular momentum. In the two flavor case, an additional contribution arises due to gapless quarks and electrons, which is responsible for Debye screening effects. We also discuss the low energy effective theory for the three flavor case in the presence of electromagnetic interactions.
\end{abstract}

DOI: 10.1103/PhysRevD.64.094013 PACS number(s): 12.38.Mh, 13.40. $-\mathrm{f}, 24.85 .+\mathrm{p}, 26.60 .+\mathrm{c}$

\section{INTRODUCTION}

Quantum chromodynamics (QCD) in the regime of high baryonic density and low temperature displays the phenomenon of color superconductivity [1-4] (see [5] for a recent review). This is a consequence of Cooper's theorem, which states that an attractive interaction close to the Fermi surface makes the system unstable toward the formation of condensates. In QCD the attractive interaction is provided by onegluon exchange between quarks in a color antisymmetric channel. The existence of the condensates leads to energy gaps for both the fermionic quasiparticles and some or all gluons. It is expected that a color superconducting state of matter could exist in the core of compact stars. Some basic properties of very dense stellar objects, such as neutron stars, may be understood through the study of cold and dense quark matter.

At asymptotically high baryonic density, and because of the asymptotic freedom of QCD, it is possible to compute the quark gap and the gluon masses from first principles [6-11]. At present, it is unclear to what extent these results can be extended to more moderate values of the baryonic density, where non-perturbative effects may become relevant [12]. Furthermore, there are no reliable methods available to perform numerical simulations of QCD at finite chemical potential. Therefore, results obtained at asymptotically large baryonic densities cannot be compared with those for less dense systems. In this light, it is most important to provide a sound understanding of the weak coupling regime. We expect that these studies lead to both the qualitatively and probably also the semiquantitatively correct behavior of the color superconductor for densities attained in the core of neutron stars.

The diquark condensates of a color superconductor break spontaneously, partially or totally, the non-Abelian gauge

\footnotetext{
*Email address: Daniel.Litim@cern.ch

†Email address: Cristina.Manuel@cern.ch
}

symmetry, depending on the number of quark flavors participating in the condensation. For two light quark flavors (2SC phase), the color group $S U(3)_{c}$ is broken down to an $S U(2)_{c}$ subgroup [3]. Five gluons acquire masses through the Anderson-Higgs mechanism, while three gluons remain massless. Also, not all quarks attain a gap. For three light quark flavors the condensates lock both color and flavor symmetry transformations [color-flavor locking (CFL) phase] [4]. The CFL condensates spontaneously break both color and flavor symmetries

$$
S U(3)_{c} \otimes S U(3)_{L} \otimes S U(3)_{R} \otimes U(1)_{B} \rightarrow S U(3)_{c+L+R} \otimes \mathcal{Z}_{2} .
$$

All gluons acquire masses in this case, and all quarks attain a gap. Furthermore, there is a Goldstone boson associated with the breaking of baryon number, and eight Goldstone bosons associated with the breaking of chiral symmetry.

The infrared physics in a color superconductor is dominated by its light degrees of freedom. For the two-flavor color superconductor, those are the unbroken gauge fields and the gapless quarks. An effective theory for the long distance physics of the $S U(2)_{c}$ gauge fields has recently been discussed for vanishing [14] and non-vanishing temperature [15]. For the CFL color superconductor the light modes are the Goldstone bosons associated with the breaking of the global symmetries. At low energies an effective Lagrangian and chiral perturbation theory can be used to study the dynamics of these light modes [16-24].

The long distance physics in a color superconductor is modified if one includes electromagnetic interactions [4,25]. Both the CFL and 2SC diquark condensates spontaneously break the standard electromagnetic symmetry. However, a linear combination of the original photon and a gluon remains massless in both cases. This new field plays the role of the "in-medium" photon in the superconductor.

In the present article, we compute the photon self-energy for the CFL and 2SC phases at very large densities, in the weak coupling regime. We show how the quark condensates affect the propagation of the "rotated" photon. In the litera- 
ture, it has sometimes been assumed that the photon propagates as in the vacuum, or as in a dense medium without Cooper pairs of quarks. We will see that none of these assumptions holds true. Our results are, therefore, relevant for a number of properties of color superconducting quark matter. Let us mention two examples. The first one concerns the charged pions and kaons of the CFL phase which acquire a mass of electromagnetic origin even in the chiral limit $m_{q}$ $=0$, as first pointed out in [26]. A computation of this electromagnetic mass, which requires the evaluation of a threeloop diagram with dressed photon propagators, is still missing, although a rough estimate was given in [24]. A second example concerns transport properties of superconducting quark matter. Knowing the infrared behavior of the electromagnetic interactions is essential for a reliable computation of transport coefficients such as viscosities and electrical and thermal conductivities of the superconducting medium.

The paper is organized as follows. In Sec. II, we study the photon self-energy for the three flavor color superconductor. We review the symmetry breaking pattern induced by the diquark condensates in a three-flavor color superconductor. Using a non-linear framework, the unbroken $U(1)$ symmetry and the corresponding in-medium photon are identified. We then study how the rotated photon couples to all the charged particles of the system (Sec. II A). The Feynman rules for quark propagators and vertices are given (Sec. II B), and the quark contribution to the one-loop photon self-energy is computed (Sec. II C and Sec. II D). We discuss the photon self-energy in the infrared region and emphasize the most relevant modifications due to the diquark condensates on the propagation properties of the photon (Sec. II E). The low energy effective theory of the CFL phase, including electromagnetic interactions, is constructed (Sec. II F). We close with a discussion of the main physical picture of photon propagation in the CFL phase (Sec. II G). In Sec. III we repeat the same analysis for the two-flavor color superconductor. We begin with a discussion of the in-medium photon and its couplings to all charged particles in a two-flavor color superconductor (Sec. III A). The Feynman rules for quark propagators and vertices are given (Sec. III B), and the computation of the quark contribution to the one-loop photon self-energy is performed (Sec. III C). The low-momentum limit of the self-energy is studied (Sec. III D), and the main physical picture of photon propagation in the 2SC phase is discussed (Sec. III E). Section IV closes with a summary of the results and an outlook. In the Appendix, we compute the contribution to the self-energy due to charged pions and kaons in the CFL phase. It is shown that charged mesons are negligible for the photon self-energy in the infrared limit. Throughout we work with natural units, $\hbar=c=k_{B}=1$.

\section{PHOTON SELF-ENERGY IN THE THREE-FLAVOR COLOR SUPERCONDUCTOR}

\section{A. Electromagnetic interactions}

The ground state of QCD at high baryonic density with three light quark flavors is described by the (spin zero) condensates [4]

$$
\left\langle q_{L}^{a i} q_{L}^{b j}\right\rangle=-\left\langle q_{R}^{a i} q_{R}^{b j}\right\rangle=k_{1} \delta_{i}^{a} \delta_{j}^{b}+k_{2} \delta_{j}^{a} \delta_{i}^{b},
$$

where $q_{L / R}$ are Weyl spinors (a sum over spinor indices is understood), and $a, b$ and $i, j$ denote flavor and color indices, respectively. These CFL condensates spontaneously break color, chiral and baryon number symmetries. As a result, all the gluons become massive through the Anderson-Higgs mechanism, while there are nine Goldstone bosons associated with the breaking of the global symmetries.

The diquark condensates also spontaneously break the standard electromagnetic symmetry. However, a combination of the electromagnetic generator and an $S U(3)$ generator leaves the CFL ground state invariant [4]. Thus, a linear combination of the original photon and a gluon remains massless and plays the role of the "new" photon in the color superconductor.

In order to explicitly identify the massless linear combination of gluon and photon it is very convenient to use the non-linear framework [16]. One first introduces left-handed and right-handed transforming fields

$$
L^{a i} \sim \epsilon^{i j k} \epsilon^{a b c}\left\langle q_{L}^{b j} q_{L}^{c k}\right\rangle^{*}, \quad R^{a i} \sim \epsilon^{i j k} \epsilon^{a b c}\left\langle q_{R}^{b j} q_{R}^{c k}\right\rangle^{*} .
$$

These fields contain the would-be Goldstone bosons which are to be absorbed by the gluons through the Higgs mechanism, as well as the Goldstone bosons associated with chiral symmetry breaking. Under

$$
S U(3)_{c} \otimes S U(3)_{L} \otimes S U(3)_{R} \otimes U(1)_{\text {e.m. }} .
$$

they transform as

$$
L \rightarrow U_{1} U_{L} L U_{c}^{\dagger}, \quad R \rightarrow U_{1} U_{R} R U_{c}^{\dagger} .
$$

Therefore, the covariant derivative acting on these fields is

$$
D_{\mu} L=\partial_{\mu} L-i e Q A_{\mu} L-i g G_{\mu}^{n} L T^{n},
$$

where $Q=\operatorname{diag}(2 / 3,-1 / 3,-1 / 3)$ is the quark charge matrix, $e$ and $g$ are the electromagnetic and strong coupling constants, and $T^{n}$ are the $S U(3)$ generators. The covariant derivative acting on $R$ acts in the same way as for $L$. The kinetic term

$$
\operatorname{Tr}\left(D_{\mu} L^{\dagger} D^{\mu} L\right)+\operatorname{Tr}\left(D_{\mu} R^{\dagger} D^{\mu} R\right)
$$

then tells us which are the massive and massless gauge eigenstates in the theory. The diagonalization of the gauge mass matrix simplifies upon replacing the standard $S U(3)$ generators by $T^{8}=(\sqrt{3} / 2) Q$ and $T^{3}=\operatorname{diag}(0,1 / 2,-1 / 2)$.

As a result, the gluons $G_{\mu}^{n}$ with $n=1, \ldots, 7$ are all massive. Their masses, which have been computed from QCD $[17,28]$, are of the order $\sim g \mu$, with $\mu$ the chemical potential. In addition, there is a combination of gluon and photon, $\widetilde{G}^{8}$, which is massive, while the orthogonal combination, $\widetilde{A}$, is massless $[25,16,29,27]:^{1}$

\footnotetext{
${ }^{1}$ The mixing angle of [25] differs from ours due to a difference in the normalization of the $S U(3)$ generators.
} 


$$
\begin{aligned}
& \widetilde{G}_{\mu}^{8}=\cos \theta_{\mathrm{CFL}} G_{\mu}^{8}+\sin \theta_{\mathrm{CFL}} A_{\mu}, \\
& \widetilde{A}_{\mu}=-\sin \theta_{\mathrm{CFL}} G_{\mu}^{8}+\cos \theta_{\mathrm{CFL}} A_{\mu} .
\end{aligned}
$$

Here, the rotation angle $\theta_{\mathrm{CFL}}$ is defined as

$$
\cos \theta_{\mathrm{CFL}}=\frac{\sqrt{3} g}{\sqrt{3 g^{2}+4 e^{2}}}, \quad \sin \theta_{\mathrm{CFL}}=\frac{2 e}{\sqrt{3 g^{2}+4 e^{2}}} .
$$

The field $\widetilde{A}$ plays the role of the "rotated" or in-medium photon in the superconductor. In contrast to the other gluons, the mass of $\widetilde{G}_{\mu}^{8}$ is of the order $\sim \sqrt{g^{2}+\frac{4}{3} e^{2}} \mu$.

We now turn to the charge eigenstates and their coupling to the in-medium photon. For the quark matter fields the Lagrangian reads

$$
\mathcal{L}_{\text {quarks }}^{\text {e.m. }}=\bar{\psi} i \widetilde{D}_{\mu} \gamma^{\mu} \psi=\bar{\psi}\left(i \partial_{\mu}-\widetilde{e} \widetilde{Q} \widetilde{A}_{\mu}\right) \gamma^{\mu} \psi,
$$

where we have introduced the gauge coupling of the inmedium photon $\widetilde{e}=e \cos \theta_{\mathrm{CFL}}$. The charge matrix $\widetilde{Q}$ is a matrix in flavor $_{(3 \times 3)} \otimes \operatorname{color}_{(3 \times 3)}$ space

$$
\widetilde{Q}=Q \otimes 1-1 \otimes Q .
$$

The linear combinations

$$
G_{\mu}^{ \pm} \equiv \frac{1}{\sqrt{2}}\left(G_{\mu}^{4} \mp i G_{\mu}^{5}\right), \quad H_{\mu}^{ \pm} \equiv \frac{1}{\sqrt{2}}\left(G_{\mu}^{6} \mp i G_{\mu}^{7}\right)
$$

are $\widetilde{Q}$-charge eigenstates with charge $\pm \widetilde{e}$. They remain mass eigenstates, because the first seven gluons have equal masses anyway. Taking into account the values of the total antisymmetric constants of $S U(3), f^{458}=f^{678}=\sqrt{3} / 2$, we find the coupling of the charged gluons to the new photon:

$$
\mathcal{L}_{\text {gluons }}^{\text {e.m. }}=\frac{1}{2} \widetilde{D}_{[\mu} G_{\nu]}^{+} \widetilde{D}_{[\mu} G_{\nu]}^{-}+\frac{1}{2} \widetilde{D}_{[\mu} H_{\nu]}^{+} \widetilde{D}_{[\mu} H_{\nu]}^{-} .
$$

Here, $\widetilde{D}_{\mu} X^{ \pm} \equiv\left(\partial_{\mu} \pm i \widetilde{e} \widetilde{A}_{\mu}\right) X^{ \pm}, X=G$ or $H$, and $A_{[\mu} B_{\nu]}$ $\equiv A_{\mu} B_{\nu}-A_{\nu} B_{\mu}$. Notice that in Eq. (2.11) we have written only the part of the Lagrangian that contains the coupling with the new photon while omitting the self-interactions among gluons, or the mass terms. The mass terms can be read off from Eq. (2.5).

Finally, the charged pions and kaons also couple to the new photon:

$$
\begin{aligned}
\mathcal{L}_{\mathrm{GB}}^{\text {e.m. }}= & \widetilde{D}_{0} \pi^{+} \widetilde{D}_{0} \pi^{-}-v_{\pi}^{2} \widetilde{D}_{i} \pi^{+} \widetilde{D}_{i} \pi^{-}+\widetilde{D}_{0} K^{+} \widetilde{D}_{0} K^{-} \\
& -v_{\pi}^{2} \widetilde{D}_{i} K^{+} \widetilde{D}_{i} K^{-},
\end{aligned}
$$

where $v_{\pi}=1 / \sqrt{3}$ is the meson velocity [17]. Electromagnetic effects also generate a mass term for the charged pions and kaons, even in the chiral limit $m_{q}=0$, although its precise value is yet unknown.
TABLE I. $\widetilde{Q}$ charges of gluons in the CFL phase, and in units of $\widetilde{e}=e \cos \theta_{\mathrm{CFL}}$.

\begin{tabular}{cccccccccc}
\hline \hline & $G_{\mu}^{1}$ & $G_{\mu}^{2}$ & $G_{\mu}^{3}$ & $G_{\mu}^{+}$ & $G_{\mu}^{-}$ & $H_{\mu}^{+}$ & $H_{\mu}^{-}$ & $\widetilde{G}_{\mu}^{8}$ \\
\hline$\widetilde{Q}$ charge & 0 & 0 & 0 & & 1 & -1 & 1 & -1 & 0 \\
\hline \hline
\end{tabular}

Summarizing, under the rotated electromagnetism four gluons, four Goldstone bosons, and four quarks are charged, as can be read off from the electromagnetic part of the Lagrangian (cf. Tables I, II and III). Their charges are integral multiples of the electron charge $\widetilde{e}$. More explicitly, in the quark sector, the up quarks of fundamental colors 2 and 3 carry charge $\widetilde{e}$, while the down and strange quarks of fundamental color 1 carry charge $-\widetilde{e}$. The remaining quarks are electrically neutral. The condensates are also neutral, as expected, as the up quark of color 2 pairs only with the down quark of color 1 , while the up quark of color 3 pairs only with the strange quark of color 1. Quark matter in the CFL phase is then $\widetilde{Q}$ neutral, as in the spectrum there are the same number of particles with positive charge $\widetilde{e}$ and negative charge $-\widetilde{e}$. It has also been argued that quark matter is $Q$ neutral [30], even in the presence of a non-vanishing and small strange quark mass and/or strange chemical potential. In this case, no further charge carriers (like electrons) are needed to make the system electrically neutral.

In order to compute the one-loop photon self-energy, it is necessary to consider all one-loop diagrams with two external photon lines and internal loops of charged particles. These are electrically charged quarks, pions, kaons, or gluons. In addition, we have to add a gauge fixing term and consider the contributions from the ghost fields. The gluons and ghosts contribution lead to a renormalization of the gauge coupling constant, in full analogy to the contribution to the one-loop photon self-energy of the gauge sector of the Standard Model in vacuum as arising from the $W_{\mu}^{ \pm}$bosons and ghosts. Since the gluons are heavy, it is easy to see that the finite part arising from the gauge boson loops is suppressed in the infrared limit. In contrast to the gluons, the charged pions and kaons are light. Their contribution to the photon polarization tensor is analogous to the one in vacuum. Apart from a renormalization of the coupling constant, they contribute a finite piece to the photon self-energy. In the Appendix, it is shown that their finite contribution is negligible in the infrared limit. Hence, polarization effects of the photon are strongly dominated in the infrared limit by pure medium effects arising from the diquark condensate. In the following sections we present in full detail the computation of the polarization tensor due to the quarks.

TABLE II. $\widetilde{Q}$ charges of pseudo Nambu-Goldstone bosons in the CFL phase.

\begin{tabular}{lcccccccc}
\hline \hline & $\pi^{+}$ & $\pi^{-}$ & $\pi^{0}$ & $K^{+}$ & $K^{-}$ & $K^{0}$ & $\bar{K}^{0}$ & $\eta$ \\
\hline$\widetilde{Q}$ charge & 1 & -1 & 0 & 1 & -1 & 0 & 0 & 0 \\
\hline \hline
\end{tabular}


TABLE III. $\widetilde{Q}$ charges of quarks in the CFL phase.

\begin{tabular}{lccccccccc}
\hline \hline & & Up & \multicolumn{1}{c}{ Down } & \multicolumn{4}{c}{ Strange } \\
Color & 1 & 2 & 3 & 1 & 2 & 3 & 1 & 2 & 3 \\
\hline$\widetilde{Q}$ charge & 0 & 1 & 1 & -1 & 0 & 0 & -1 & 0 & 0 \\
\hline \hline
\end{tabular}

\section{B. Feynman rules}

The computation of the one-loop photon self-energy will be done in Euclidean space-time, using the imaginary time formalism. We first discuss the Feynman rules for the quark propagators and vertices. These have a complicated structure, as they mix both color and flavor quark indices. It thus proves convenient to work in a quark basis in which the quark propagator simplifies. [17]

We define the color-flavor basis by the transformation

$$
\psi_{a i}=\frac{1}{\sqrt{2}} \sum_{A=1}^{9} \lambda_{a i}^{A} \psi^{A}
$$

where $a$ and $i$ refer to the quark flavor and color indices, respectively, and $\lambda^{A}$, for $A=1, \ldots, 8$ are the Gell-Mann matrices, while $\lambda^{9}=\sqrt{\frac{2}{3}} 1$. The gap matrix can be diagonalized as

$$
\Delta^{A B}=\delta^{A B} \Delta^{A}
$$

where $\Delta^{A}=k_{2}$ for $A=1, \ldots, 8$ denotes the octet gap, and $\Delta^{9}=k_{2}+3 k_{1}$ the singlet gap.

We will use the Nambu-Gorkov formalism in the computation. The Nambu-Gorkov fields are defined as

$$
\Psi=\left(\begin{array}{c}
\psi \\
\psi_{c}
\end{array}\right), \quad \bar{\Psi}=\left(\bar{\psi}, \bar{\psi}_{c}\right)
$$

where $\psi(x)$ is a Dirac spinor, while $\psi_{c}(x)=C \bar{\psi}^{T}(x)$ is the charge-conjugate spinor.

In the CFL basis the Nambu-Gorkov matrix propagator becomes diagonal in the $A$ index,

$$
S_{A B}(K)=\delta_{A B}\left(\begin{array}{cc}
S_{A}^{+}(K) & \Xi_{A}^{-}(K) \\
\Xi_{A}^{+}(K) & S_{A}^{-}(K)
\end{array}\right),
$$

where $K=\left(k_{0}, \mathbf{k}\right)$ is the four-momentum, and $k_{0}=-i \omega_{n}=$ $-i(2 n+1) \pi T$ is a fermionic Matsubara frequency. For massless quarks,

$$
\begin{aligned}
& S_{A}^{ \pm}(K)=\frac{\Lambda_{\mathbf{k}}^{ \pm} \gamma^{0}\left(k_{0} \mp \mu \pm k\right)}{k_{0}^{2}-\left(E_{k}^{A}\right)^{2}}+\frac{\Lambda_{\mathbf{k}}^{\mp} \gamma^{0}\left(k_{0} \mp \mu \mp k\right)}{k_{0}^{2}-\left(\bar{E}_{k}^{A}\right)^{2}}, \\
& \Xi_{A}^{+}(K)=\gamma_{5}\left\{\frac{\Lambda_{\mathbf{k}}^{-} \Delta^{A}}{k_{0}^{2}-\left(E_{k}^{A}\right)^{2}}+\frac{\Lambda_{\mathbf{k}}^{+} \bar{\Delta}^{A}}{k_{0}^{2}-\left(\bar{E}_{k}^{A}\right)^{2}}\right\},
\end{aligned}
$$

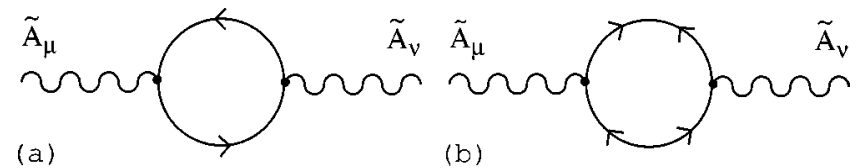

FIG. 1. Quark contributions to the 1-loop photon self-energy in the superconducting phase.

$$
\Xi_{A}^{-}(K)=-\gamma_{5}\left\{\frac{\Lambda_{\mathbf{k}}^{+}\left(\Delta^{A}\right)^{*}}{k_{0}^{2}-\left(E_{k}^{A}\right)^{2}}+\frac{\Lambda_{\mathbf{k}}^{-}\left(\bar{\Delta}^{A}\right)^{*}}{k_{0}^{2}-\left(\bar{E}_{k}^{A}\right)^{2}}\right\},
$$

where $k=|\mathbf{k}|$, and

$$
\Lambda_{\mathbf{k}}^{ \pm}=\frac{1 \pm \gamma_{0} \gamma \cdot \hat{\mathbf{k}}}{2}
$$

are the positive/negative energy projectors. The energies of particles, $E$, and antiparticles, $\bar{E}$, read

$$
E_{k}^{A}=\sqrt{\xi_{k}^{2}+\left(\Delta^{A}\right)^{2}}, \quad \bar{E}_{k}^{A}=\sqrt{\overline{\xi_{k}^{2}+\left(\bar{\Delta}^{A}\right)^{2}}},
$$

where in the above dispersion relations $\xi_{k}=\mu-k, \bar{\xi}_{k}=\mu$ $+k$, and $\Delta^{A}$ and $\bar{\Delta}^{A}$ are the gap and the antigap, respectively.

From Eq. (2.8) we can obtain the quark-photon interaction in the CFL basis:

$$
\frac{\widetilde{e}}{2} \sum_{B, C=1}^{9}\left[\operatorname{Tr}\left(\lambda^{B} Q \lambda^{C}\right)-\operatorname{Tr}\left(\lambda^{C} Q \lambda^{B}\right)\right] \bar{\psi}^{B} \gamma^{\mu} \widetilde{A}_{\mu} \psi^{C} .
$$

For the complex conjugate fields the quark-photon interaction term becomes

$$
-\frac{\tilde{e}}{2} \sum_{B, C=1}^{9}\left[\operatorname{Tr}\left(\lambda^{C} Q \lambda^{B}\right)-\operatorname{Tr}\left(\lambda^{B} Q \lambda^{C}\right)\right] \bar{\psi}_{c}^{B} \gamma^{\mu} \widetilde{A}_{\mu} \psi_{c}^{C} .
$$

These terms allow us to define the Feynman rules for the quark-photon vertices in the CFL basis.

\section{Traces in color-flavor space}

To one-loop order, the two diagrams given in Fig. 1 have to be evaluated. The full line in Fig. 1(a) [Fig. 1(b)] denotes the $S_{A}^{ \pm}$part ( $\Xi_{A}^{ \pm}$part) of the quark propagator in the NambuGorkov representation [cf. Eq. (2.16)].

Using the Feynman rules of the previous subsection one gets

$$
\begin{aligned}
\Pi^{\mu \nu}(P)= & \frac{\widetilde{e}^{2}}{8} \sum_{B, C=1}^{9}\left[\operatorname{Tr}\left(\lambda^{B} Q \lambda^{C}\right)-\operatorname{Tr}\left(\lambda^{C} Q \lambda^{B}\right)\right] \\
& \times\left[\operatorname{Tr}\left(\lambda^{C} Q \lambda^{B}\right)-\operatorname{Tr}\left(\lambda^{B} Q \lambda^{C}\right)\right] I_{B C}^{\mu \nu}(P) \\
& -\frac{\widetilde{e}^{2}}{8} \sum_{B, C=1}^{9}\left[\operatorname{Tr}\left(\lambda^{B} Q \lambda^{C}\right)-\operatorname{Tr}\left(\lambda^{C} Q \lambda^{B}\right)\right] \\
& \times\left[\operatorname{Tr}\left(\lambda^{B} Q \lambda^{C}\right)-\operatorname{Tr}\left(\lambda^{C} Q \lambda^{B}\right)\right] R_{B C}^{\mu \nu}(P),
\end{aligned}
$$


where

$$
\begin{aligned}
& I_{B C}^{\mu \nu}(P)=\sum_{e= \pm} \int \frac{d^{4} K}{(2 \pi)^{4}} \operatorname{Tr}\left[\gamma^{\mu} S_{C}^{e}(K) \gamma^{\nu} S_{B}^{e}(K-P)\right], \\
& R_{B C}^{\mu \nu}(P)=\sum_{e= \pm} \int \frac{d^{4} K}{(2 \pi)^{4}} \operatorname{Tr}\left[\gamma^{\mu} \Xi_{C}^{e}(K) \gamma^{\nu} \Xi_{B}^{-e}(K-P)\right] .
\end{aligned}
$$

Notice that above we used the standard notation of the imaginary time formalism, so that for $T \neq 0$

$$
\int \frac{d^{4} K}{(2 \pi)^{4}} \equiv T \sum_{n=-\infty}^{n=\infty} \int \frac{d^{3} k}{(2 \pi)^{3}},
$$

where the sum is over the Matsubara frequencies.

Now the $U(3)$ color-flavor traces in Eq. (2.22) can be easily performed, just by realizing that the quark propagators $S_{A}^{ \pm}$and $\Xi_{A}^{ \pm}$are all the same for the octet, $A=1, \ldots, 8$, while they differ for the singlet $A=9$. In particular, the relevant traces are

$$
\begin{aligned}
& \sum_{B, C=1}^{9} \operatorname{Tr}\left(\lambda^{B} Q \lambda^{C}\right) \operatorname{Tr}\left(\lambda^{C} Q \lambda^{B}\right) M_{B C} \\
& \quad=\frac{8}{9}\left(7 M_{11}+M_{19}+M_{91}\right), \\
& \sum_{B, C=1}^{9} \operatorname{Tr}\left(\lambda^{B} Q \lambda^{C}\right) \operatorname{Tr}\left(\lambda^{B} Q \lambda^{C}\right) M_{B C} \\
& \quad=\frac{8}{9}\left(-2 M_{11}+M_{19}+M_{91}\right),
\end{aligned}
$$

where $M_{B C}$ can be either $I_{B C}$ or $R_{B C}$. In the evaluation, we made use of $Q=\frac{1}{2}\left(\lambda^{3}+\lambda^{8} / \sqrt{3}\right)$. Finally, this leads to

$$
\Pi^{\mu \nu}(P)=2 \widetilde{e}^{2}\left[I_{11}^{\mu \nu}(P)+R_{11}^{\mu \nu}(P)\right] .
$$

It is curious to note that the singlet quark propagators do not participate in the photon self-energy. This is quite different from what happens in the gluon self-energy in the CFL phase, where the one-loop terms contain contributions from both octet quark propagators, and also from a mixing between singlet and octet quark propagators $[17,28,24]$.

\section{Sum over Matsubara frequencies}

After the explicit evaluation of the spinor traces and the sum over Matsubara frequencies in Eqs. (2.23), we find an expression for the one-loop photon polarization tensor. Since the photon polarization tensor Eq. (2.27) depends only on the octet gap $\Delta^{1}$, but not on the singlet gap $\Delta^{9}$, we denote the former as $\Delta \equiv \Delta^{1}$ from now on. We also drop the octet index for the energies, $E \equiv E^{1}$, and $\bar{E} \equiv \bar{E}^{1}$. More explicitly, we find

$$
\begin{aligned}
\Pi^{00}(P)= & -\frac{c \widetilde{e}^{2}}{2} \int \frac{d^{3} k}{(2 \pi)^{3}} \sum_{e_{1}, e_{2}= \pm}\left(1+e_{1} e_{2} \hat{\mathbf{k}}_{1} \cdot \hat{\mathbf{k}}_{2}\right) \\
& \times\left[\left(\frac{1}{p_{0}+E_{1}+E_{2}}-\frac{1}{p_{0}-E_{1}-E_{2}}\right)\left(1-N_{1}-N_{2}\right)\right. \\
& \times \frac{E_{1} E_{2}-\xi_{1} \xi_{2}-\Delta_{1} \Delta_{2}}{2 E_{1} E_{2}}+\left(\frac{1}{p_{0}-E_{1}+E_{2}}\right. \\
& \left.\left.+\frac{1}{p_{0}+E_{1}-E_{2}}\right)\left(N_{1}-N_{2}\right) \frac{E_{1} E_{2}+\xi_{1} \xi_{2}+\Delta_{1} \Delta_{2}}{2 E_{1} E_{2}}\right],
\end{aligned}
$$

$$
\begin{aligned}
\Pi^{0 i}(P)= & -\frac{c \widetilde{e}^{2}}{2} \int \frac{d^{3} k}{(2 \pi)^{3}} \sum_{e_{1}, e_{2}= \pm}\left(e_{1} \hat{k}_{1}^{i}+e_{2} \hat{k}_{2}^{i}\right) \\
& \times\left[\left(\frac{1}{p_{0}+E_{1}+E_{2}}-\frac{1}{p_{0}-E_{1}-E_{2}}\right)\left(1-N_{1}-N_{2}\right)\right. \\
& \times\left(\frac{\xi_{2}}{2 E_{2}}-\frac{\xi_{1}}{2 E_{1}}\right)+\left(\frac{1}{p_{0}-E_{1}+E_{2}}+\frac{1}{p_{0}+E_{1}-E_{2}}\right) \\
& \left.\times\left(N_{1}-N_{2}\right)\left(\frac{\xi_{2}}{2 E_{2}}+\frac{\xi_{1}}{2 E_{1}}\right)\right], \\
\Pi^{i j}(P)= & -\frac{c \widetilde{e}^{2}}{2} \int \frac{d^{3} k}{(2 \pi)^{3}} e_{1}, e_{2}= \pm \\
& \left.+e_{1} e_{2}\left(\hat{k}_{1}^{i} \hat{k}_{2}^{j}+\hat{k}_{1}^{j} \hat{k}_{2}^{i}\right)\right] \times\left[\left(\frac{1}{p_{0}+E_{1}+E_{2}}\right.\right. \\
& \left.-\frac{1}{p_{0}-E_{1}-E_{2}}\right)\left(1-e_{1} e_{2} \hat{\mathbf{k}}_{1} \cdot \hat{\mathbf{k}}_{2}\right) \\
& \times \frac{E_{1} E_{2}-\xi_{1} \xi_{2}+\Delta_{1} \Delta_{2}}{2 E_{1} E_{2}}+\left(\frac{1}{p_{0}-E_{1}+E_{2}}\right. \\
& \left.\left.+\frac{1}{p_{0}+E_{1}-E_{2}}\right)\left(N_{1}-N_{2}\right) \frac{E_{1} E_{2}+\xi_{1} \xi_{2}-\Delta_{1} \Delta_{2}}{2 E_{1} E_{2}}\right],
\end{aligned}
$$

where $N_{i}=1 /\left[\exp \left(E_{i} / T\right)+1\right]$ is the fermionic distribution function. The coefficient

$$
c=\sum_{\text {gapped }}\left(\frac{\tilde{q}_{i}}{\widetilde{e}}\right)^{2}
$$

counts the square of the charges $\tilde{q}_{i}$ of all gapped quarks, in units of $\widetilde{e}$. In the CFL phase, $c=4$. The condensed notation of Eqs. (2.28) is borrowed from Ref. [13] and goes as follows. The indices $e_{i}$ take into account the contribution of particles $\left(e_{i}=+\right)$ and antiparticles $\left(e_{i}=-\right)$. All other quantities that carry a subscript " 1 " or " 2 " have to be evaluated at the corresponding value of the index $e_{1}$ or $e_{2}$, and for the corresponding momenta $\mathbf{k}_{1} \equiv \mathbf{k}$ or $\mathbf{k}_{2} \equiv \mathbf{k}-\mathbf{p}$. For example, if $e_{2}=-$, then $E_{2} \equiv \sqrt{\bar{\xi}_{k-p}^{2}+\bar{\Delta}^{2}}$. 
It is worth emphasizing that the structure of the polarization tensor found in Eqs. (2.28) is very similar to the structure of the polarization tensor of the unbroken $S U(2)_{c}$ gauge fields in the 2SC phase, as computed in Ref. [13]. It suffices to change the coupling constant and the numerical prefactor $c \widetilde{e}^{2} \rightarrow \frac{1}{2} N_{f} g^{2}$ to obtain the expressions given in Eqs. (99) of Ref. [13], where $N_{f}$ is the number of quark flavors, and the factor $\frac{1}{2}$ arises from the trace of the $S U(3)$ generators in the fundamental representation.

\section{E. Photon self-energy at low momentum}

After analytical continuation to Minkowski space-time, one can study the behavior of the self-energy for different values of the external frequency and momentum. Since the behavior of the polarization tensor for the unbroken $S U(2)_{c}$ subgroup of the 2SC phase has been studied in the literature, we will not present a detailed analysis here. We refer to the literature $[13,14,31]$ for more explicit details.

Let us first define the longitudinal and transverse parts of the photon polarization tensor in the usual manner,

$$
\begin{aligned}
\Pi^{00}\left(p_{0}, \mathbf{p}\right)= & \Pi_{L}\left(p_{0}, \mathbf{p}\right), \\
\Pi^{0 i}\left(p_{0}, \mathbf{p}\right)= & \frac{p_{0}}{p} \frac{p^{i}}{p} \Pi_{L}\left(p_{0}, \mathbf{p}\right), \\
\Pi^{i j}\left(p_{0}, \mathbf{p}\right)= & \left(\delta^{i j}-\frac{p^{i} p^{j}}{p^{2}}\right) \Pi_{T}\left(p_{0}, \mathbf{p}\right) \\
& +\frac{p^{i} p^{j}}{p^{2}} \frac{p_{0}^{2}}{p^{2}} \Pi_{L}\left(p_{0}, \mathbf{p}\right),
\end{aligned}
$$

Below, we will discuss only the zero temperature case. As expected, there is no Debye or Meissner screening for the photon at vanishing temperature, because

$$
\begin{aligned}
& \lim _{p \rightarrow 0} \Pi^{00}\left(p_{0}=0, \mathbf{p}\right)=0, \\
& \Pi^{0 i}\left(p_{0}=0, \mathbf{p}\right)=\Pi^{i j}\left(p_{0}=0, \mathbf{p}\right)=0 .
\end{aligned}
$$

In the infrared limit the photon self-energy is dominated by the quark contribution (cf. the Appendix). For $p_{0}, p \ll \Delta$, it is possible to compute the value of the polarization tensor (2.28). Expanding the polarization tensor to quadratic order in $p_{0}$ and $p$, and taking the limit of very large density, one finds

$$
\begin{aligned}
& \Pi_{L}\left(p_{0}, \mathbf{p}\right)=-\tilde{\kappa} p^{2}, \\
& \Pi_{T}\left(p_{0}, \mathbf{p}\right)=-\tilde{\kappa} p_{0}^{2},
\end{aligned}
$$

where

$$
\tilde{\kappa}=\frac{c}{18 \pi^{2}} \frac{\widetilde{e}^{2} \mu^{2}}{\Delta^{2}}
$$

The coefficient $c$ has been defined in Eq. (2.29); $c=4$ in the CFL phase.

The presence of the quark condensate modifies the photon dispersion relations at low momenta. The dielectric constant of the CFL medium becomes

$$
\tilde{\epsilon}=1+\tilde{\kappa}=1+\frac{2}{9 \pi^{2}} \frac{\widetilde{e}^{2} \mu^{2}}{\Delta^{2}}
$$

while the magnetic susceptibility $\tilde{\lambda}=1$ remains unchanged to leading order. This is due to the fact that the CFL condensates have zero spin and angular momentum, and hence a vanishing magnetic moment. The velocity $v$ of the $\widetilde{Q}$ photon is given through $v^{2}=1 / \tilde{\epsilon} \tilde{\lambda}$. In the limit of asymptotically high densities, the gap is exponentially suppressed $\Delta$ $\sim \mu g^{-5} \exp \left(-3 \pi^{2} / \sqrt{2} g\right)$ [6], and therefore $\tilde{\kappa} \gg 1$ and $\tilde{\epsilon} \gg 1$. As a consequence, the photon velocity is very much suppressed when compared to the vacuum theory, $v \ll 1$. Furthermore, the static potential created by a test charge particle, $V(r)=\tilde{e} /\left(4 \pi^{2} \tilde{\epsilon} r\right)$, is greatly reduced with respect to the Coulomb potential in vacuum.

For external momenta in the range $\Delta \ll p_{0}, p \ll \mu$, the photon polarization tensor (2.28) reduces to the hard dense loop (HDL) limit [32,33], up to corrections of order $\tilde{e}^{2} \mu^{2} \Delta / p[13]$,

$$
\Pi^{\mu \nu}\left(p_{0}, \mathbf{p}\right)=\tilde{M}^{2}\left(-g^{\mu 0} g^{\nu 0}+p_{0} \int \frac{d \Omega_{\mathbf{v}}}{4 \pi} \frac{v^{\mu} v^{\nu}}{p_{0}-\mathbf{p} \cdot \mathbf{v}+i 0^{+}}\right),
$$

where

$$
\tilde{M}^{2}=4 \frac{\widetilde{e}^{2} \mu^{2}}{\pi^{2}}
$$

is the corresponding Debye mass. The reason that the polarization tensor reduces to the HDL result is the following [13]. For sufficiently large photon momenta, the photon wave length is sufficiently short to resolve the individual quarks within the Cooper pairs: the effects due to pairing are not visible for these modes, to leading order. Consequently, one recovers the known leading-order result of the normal phase.

The intermediate region behavior of the functions (2.28) with $\Delta<p_{0}, p \ll \mu$ has recently been studied in [31]. In this region it is possible to find analytical expressions for the imaginary part of the self-energy, which is only nonvanishing for $p_{0} \geqslant 2 \Delta$, while the real part can only be studied numerically. As a result, the photon self-energy deviates significantly from the HDL limit only for frequencies $p_{0} \sim \Delta$ [31].

If the photon momentum is not too large, $p \ll \sqrt{g} \mu$, it is possible to show that the quark contribution dominates over that due to charged pions and kaons in this momentum region (cf. the Appendix). Hence, the photon self-energy is well approximated by Eq. (2.35). 
We leave for a future project the study of electromagnetic properties of the color superconductors at non-zero temperature $T$. However, notice that the thermal contribution to the polarization tensor (2.28) for $p_{0}, p \ll \Delta$ reduces to a "hard superconducting loop" [15] and additional thermal corrections to the value of $\tilde{\kappa}$.

\section{F. Low energy effective theory for the CFL superconductor}

The low energy effective theory for a CFL superconductor, in the absence of electromagnetic interactions, has been discussed in detail in Refs. [16-24]. The physics is dominated by the light degrees of freedom, the NambuGoldstone bosons, resulting from the spontaneous breaking of chiral and baryon number symmetry. The low energy physics is modified once electromagnetism is included, because the photon field is also light. For $p_{0}, p \ll 2 \Delta$, the low energy effective theory is obtained after integrating out the heavy modes. Including electromagnetism, and in the chiral limit $m_{q}=0$, the effective Lagrangian reads

$$
\begin{aligned}
\mathcal{L}= & \frac{\tilde{\epsilon}}{2} \widetilde{\mathbf{E}} \cdot \widetilde{\mathbf{E}}-\frac{1}{2} \widetilde{\mathbf{B}} \cdot \widetilde{\mathbf{B}}+\frac{f_{\pi}^{2}}{4}\left[\operatorname{Tr}\left(\widetilde{D}_{0} \Sigma \widetilde{D}_{0} \Sigma^{\dagger}\right)\right. \\
& \left.-v_{\pi}^{2} \operatorname{Tr}\left(\widetilde{D}_{i} \Sigma \widetilde{D}_{i} \Sigma^{\dagger}\right)\right]+\widetilde{e}^{2} C \operatorname{Tr}\left(Q \Sigma Q \Sigma^{\dagger}\right),
\end{aligned}
$$

where $\widetilde{\mathbf{E}}$ and $\widetilde{\mathbf{B}}$ are the in-medium electric and magnetic fields, respectively. The unitary matrix $\Sigma$ contains the Goldstone fields, and the covariant derivative acting on $\Sigma$ is

$$
\widetilde{D}_{\mu} \Sigma=\partial_{\mu} \Sigma-i \widetilde{e} Q \widetilde{A}_{\mu} \Sigma+i \widetilde{e} \Sigma Q \widetilde{A}_{\mu} .
$$

In Eq. (2.37) we have omitted the Goldstone boson associated with the breaking of $U(1)_{B}$, as it does not couple to the photon. The values of the pion decay constant $f_{\pi}$ and the pion velocity $v_{\pi}$ at high baryonic density have been computed from the microscopic theory, finding [17-24]

$$
f_{\pi}^{2}=\frac{21-8 \ln 2}{18} \frac{\mu^{2}}{2 \pi^{2}}, \quad v_{\pi}=\frac{1}{\sqrt{3}} .
$$

In this paper we have computed the value of the dielectric constant $\tilde{\epsilon}$. The last term in Eq. (2.37) represents, to leading order, a mass term for the charged pions and kaons. This mass term is generated because the electromagnetic interactions represent an explicit breaking of chiral symmetry. The constant $C$ obeys a sum rule, but it has not yet been computed.

At higher order in an energy expansion one could also add to Eq. (2.37) the Wess-Zumino-Witten term, describing anomalous processes such as $\pi^{0} \rightarrow \tilde{\gamma} \tilde{\gamma}[27,34,35]$.

\section{G. Discussion}

We briefly discuss the main physical picture that has emerged from the present analysis. Under the rotated $U(1)$ symmetry, we have seen that the quark charges differ from the vacuum $U(1)$ electric charges. The in-medium charges are all integer and such that the diquark condensates are neu-

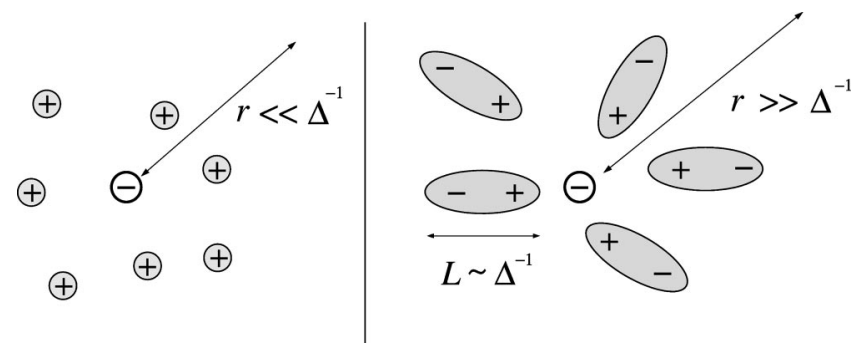

FIG. 2. Screening of a negative test charge in the CFL phase. Left panel: for length scales $r$ with $(\widetilde{e} \mu)^{-1} \sim r \ll \Delta^{-1}$, the screening is dominated by effectively ungapped charged quarks. Here, these are the up quarks of colors 2 and 3. Right panel: For length scales $r \gg \Delta^{-1}$, all quarks are bound within $\widetilde{Q}$-neutral condensates. Therefore, no Debye screening takes place. However, the condensates have an electrical dipole moment and align themselves in an electrical field. This strongly modifies the dielectric constant.

tral, although their components are not, in general. In the CFL phase, the in-medium photon propagation properties are strongly affected by the charged particles and the existence of the diquark condensates.

If the wavelength associated with the photon is smaller than the mean separation among the quarks that form the Cooper pairs, the photon propagation is not affected by the condensate. In this regime, the photon can resolve the components of the condensate. Therefore the photon self-energy matches, to leading order, the corresponding one in the nonsuperconducting phase. The electric fields acquire a Debye mass, electric and magnetic fields are Landau damped, but there is no static magnetic screening. The electric Debye mass $\widetilde{M}$ stems from all charged quarks in the CFL phase. If the photon momentum is large enough typical quantum effects that involve the creation and destruction of virtual pairs of charged pions and kaons would also modify the dielectric constant associated with the electromagnetic fields.

In turn, if the photon wavelength is large enough, larger than the mean separation among the quarks that form Cooper pairs, it can no longer resolve the components of the condensate. Consequently, and in the absence of other free charge carriers (like electrons), the long wavelength photons are not Debye screened. However, the condensates still act as electric dipoles, with total spin and angular momentum zero. This modifies the in-medium electric properties, leading to a strong increase of the dielectric constant $\tilde{\epsilon}$. The in-medium magnetic permeability remains unchanged. This is due to the fact that the condensates have vanishing magnetic moment, but would change if the condensates had a non-vanishing spin or angular momentum [8]. However, condensation in the lowest angular momentum channel is favored. We conclude that the velocity of (transverse) in-medium photons is strongly suppressed as opposed to the vacuum.

In the static limit, the resulting picture is very simple (cf. Fig. 2). Introducing a test charge in the superconductor would produce a static potential. For distances $r \sim(\widetilde{e} \mu)^{-1}$ $\ll \Delta^{-1}$, the electric test charge is screened by effectively free charge carriers; here, the up quark of colors 2 and 3. The associated static potential is given by $V(r)$ $=\widetilde{e} \exp (-\tilde{M} r) /(4 \pi r)$. At larger distances, $r \gg \Delta^{-1}$, Debye 
screening is no longer working because every positively charged quark comes bundled in Cooper pairs with a negatively charged one. The static potential produced by a test charge is $V(r)=\tilde{e} /(4 \pi \tilde{\epsilon} r)$. Because $\tilde{\epsilon} \gg 1$, the potential is greatly reduced in comparison to the Coulomb potential in vacuum.

Finally, we remark that all $\widetilde{Q}$-charged hadronic excitations in the CFL phase, and for non-vanishing quark masses, acquire a gap [4,5]. Therefore, the photon cannot scatter if its energy is below the energy of the lightest charged mode; CFL matter is thus transparent. Here, we have computed the refraction index of CFL matter with the vacuum, given by $\tilde{n}=\tilde{\epsilon}^{1 / 2}$. In the high density region, the refraction index obeys $\tilde{n} \gg 1$.

\section{PHOTON SELF-ENERGY IN THE TWO-FLAVOR COLOR SUPERCONDUCTOR}

\section{A. Electromagnetic interactions}

The (spin zero) condensates of QCD with two light quark flavors differ from those found in Eq. (2.1). In this case one finds

$$
\left\langle q_{L}^{a i} q_{L}^{b j}\right\rangle=-\left\langle q_{R}^{a i} q_{R}^{b j}\right\rangle=\epsilon^{i j 3} \epsilon_{a b} \Delta,
$$

where the gap itself picks up a direction in color space. By a global color rotation this has been fixed to be the $k=3$ direction. The original symmetries of the theory are

$$
S U(3)_{c} \otimes S U(2)_{L} \otimes S U(2)_{R} \otimes U(1)_{B},
$$

and these are broken by the condensates to $S U(2)_{C}$ $\otimes S U(2)_{L} \otimes S U(2)_{R} \otimes \widetilde{U}(1)_{B}$ [36]. The group $\widetilde{U}(1)_{B}$ corresponds to a modified global baryon symmetry, whose generator is $\widetilde{B}=B-(2 \sqrt{3} / 3) T^{8}$, where $B=\frac{1}{3} \operatorname{diag}(1,1,1)$ is the standard baryon number generator. In this section we use the standard $S U(3)$ generators, so that $T^{8}=(1 / 2 \sqrt{3}) \operatorname{diag}(1,1$, $-2)$. As a consequence of the Anderson-Higgs mechanism, five gluons acquire masses, which are of order $\sim g \mu$, although not all of them are equal [13]. Notice also that not all the quarks attain a gap. In particular, the up and down quarks of fundamental color 3 are gapless.

Exactly as it happens for the CFL superconductor, the diquark condensates (3.1) spontaneously break the standard electromagnetic symmetry. A local $U(1)$ symmetry, which is a combination of the standard electromagnetic $U(1)$ and a generator of $S U(3)_{c}$, remains unbroken. In order to identify the in-medium photon, it is convenient to use the non-linear framework. This has been done in full detail in Refs. [36,27]. The massive $\widetilde{G}^{8}$ and massless $\widetilde{A}$ fields are [29,27]

$$
\begin{aligned}
& \widetilde{G}_{\mu}^{8}=\cos \theta_{2 \mathrm{SC}} G_{\mu}^{8}+\sin \theta_{2 \mathrm{SC}} A_{\mu}, \\
& \widetilde{A}_{\mu}=-\sin \theta_{2 \mathrm{SC}} G_{\mu}^{8}+\cos \theta_{2 \mathrm{SC}} A_{\mu},
\end{aligned}
$$

where
TABLE IV. $\widetilde{Q}$ charges of gluons in the 2SC phase, and in units of $\widetilde{e}=e \cos \theta_{2 \mathrm{SC}}$.

\begin{tabular}{cccccccccc}
\hline \hline & $G_{\mu}^{1}$ & $G_{\mu}^{2}$ & $G_{\mu}^{3}$ & $G_{\mu}^{+}$ & $G_{\mu}^{-}$ & $H_{\mu}^{+}$ & $H_{\mu}^{-}$ & $\widetilde{G}_{\mu}^{8}$ \\
\hline$\widetilde{Q}$ charge & 0 & 0 & 0 & & $\frac{1}{2}$ & $-\frac{1}{2}$ & $\frac{1}{2}$ & $-\frac{1}{2}$ & 0 \\
\hline \hline
\end{tabular}

$$
\cos \theta_{2 \mathrm{SC}}=\frac{\sqrt{3} g}{\sqrt{3 g^{2}+e^{2}}}, \quad \sin \theta_{2 \mathrm{SC}}=\frac{e}{\sqrt{3 g^{2}+e^{2}}} .
$$

The field $\widetilde{A}$ is the in-medium photon. Notice that the mixing angles differ for the CFL and the 2SC superconductors.

The quark fields couple to the new photon as

$$
\mathcal{L}_{\text {quarks }}^{\text {e.m. }}=\bar{\psi} i \widetilde{D}_{\mu} \gamma^{\mu} \psi=\bar{\psi}\left(i \partial_{\mu}-\widetilde{e} \widetilde{Q} \widetilde{A}_{\mu}\right) \gamma^{\mu} \psi .
$$

The rotated gauge coupling reads $\widetilde{e}=e \cos \theta_{2 \mathrm{SC}}$, and the charge matrix $\widetilde{Q}$ is a matrix in $\operatorname{flavor}_{(2 \times 2)} \otimes \operatorname{color}_{(3 \times 3)}$ space,

$$
\widetilde{Q}=Q \otimes 1-\frac{1}{\sqrt{3}} 1 \otimes T^{8} .
$$

In the two flavor case $Q=\operatorname{diag}(2 / 3,-1 / 3)$.

Some gluons are electrically charged. Using the definitions of Eq. (2.10), we find

$$
\mathcal{L}_{\text {gluons }}^{\text {e.m. }}=\frac{1}{2} \widetilde{D}_{[\mu} G_{\nu]}^{+} \widetilde{D}_{[\mu} G_{\nu]}^{-}+\frac{1}{2} \widetilde{D}_{[\mu} H_{\nu]}^{+} \widetilde{D}_{[\mu} H_{\nu]}^{-} .
$$

Here, $\widetilde{D}_{\mu} X^{ \pm} \equiv\left[\partial_{\mu} \pm i(\widetilde{e} / 2) \widetilde{A}_{\mu}\right] X^{ \pm}, X=G$ or $H$, and $A_{[\mu} B_{\nu]}$ $\equiv A_{\mu} B_{\nu}-A_{\nu} B_{\mu}$. Hence, in the 2SC phase, and unlike the case in the CFL phase, the gauge fields $G^{ \pm}$and $H^{ \pm}$of Eq. (2.10) have the $\widetilde{Q}$ charges $\widetilde{e} / 2$. The remaining gluons are electrically neutral (cf. Table IV).

Under this rotated electromagnetism we then see that four gluons are electrically charged, but their charges are halfinteger multiples of the electron charge $\widetilde{e}$ (cf. Table IV). The up quarks of fundamental colors 1 and 2 carry charge $\widetilde{e} / 2$, while the down quarks of colors 1 and 2 carry charge $-\widetilde{e} / 2$, so that the condensates are electrically neutral. The gapless up quark of color 3 carries the charge $\widetilde{e}$, while the gapless down quark is neutral (cf. Table V).

In order to make the whole system electrically neutral one should add a background of particles with negative charges, such as strange quarks and/or electrons. The strange quarks of fundamental colors 1 and 2 carry charge $-\tilde{e} / 2$, while the strange quark of color 3 is neutral. This would be enough to make the system both $Q$ and $\widetilde{Q}$ neutral. In this section we work under the assumption that the strange quark mass is

TABLE V. $\widetilde{Q}$ charges of quarks in the 2 SC phase.

\begin{tabular}{lccccccccc}
\hline \hline & & Up & & \multicolumn{3}{c}{ Down } & \multicolumn{3}{c}{ Strange } \\
Color & 1 & 2 & 3 & 1 & 2 & 3 & 1 & 2 & 3 \\
\hline$\widetilde{Q}$ charge & $\frac{1}{2}$ & $\frac{1}{2}$ & 1 & $-\frac{1}{2}$ & $-\frac{1}{2}$ & 0 & $-\frac{1}{2}$ & $-\frac{1}{2}$ & 0 \\
\hline \hline
\end{tabular}


$m_{s} \rightarrow \infty$, in which case the strange quarks do not play a dynamical role. We will consider that there is a finite density of electrons, with an associated chemical potential $\mu_{e}$.

As in the previous section, we will compute the one-loop photon self-energy due to the quark matter sector of the theory, as we are basically interested in studying the infrared behavior of the photon self-energy.

\section{B. Feynman rules}

We use the same conventions as in the previous section, and perform the computation using the imaginary time formalism. We first give the quark propagators in the 2SC phase. The Nambu-Gorkov propagator reads [8]

$$
\mathcal{S}(K)=\left(\begin{array}{ll}
\mathcal{S}^{+}(K) & \Xi^{-}(K) \\
\Xi^{+}(K) & \mathcal{S}^{-}(K)
\end{array}\right),
$$

where every term in the matrix is also a matrix in color, flavor and spinor spaces. In particular,

$$
\begin{aligned}
& \left(\mathcal{S}^{ \pm}\right)_{i j}^{a b}(K)=\delta^{a b}\left(\delta_{i j}-\delta_{i 3} \delta_{j 3}\right) S^{ \pm}(K)+\delta^{a b} \delta_{i 3} \delta_{j 3} S_{0}^{ \pm}(K), \\
& \left(\Xi^{ \pm}\right)_{i j}^{a b}(K)= \pm \epsilon_{a b} \epsilon_{i j 3} \Xi^{ \pm}(K),
\end{aligned}
$$

where $a, b$ denote flavor indices, and $i, j$ are color indices. For massless quarks, $S^{ \pm}$and $\Xi^{ \pm}$agree with the propagators in Eqs. (2.17), after replacing the CFL gaps and antigaps by their 2SC counterparts. The free propagators $S_{0}^{ \pm}$, which describe the gapless quarks, are obtained from $S^{ \pm}$by putting $\Delta=\bar{\Delta}=0$. The quark-photon vertex follows from Eq. (3.4).

\section{Traces in color and flavor space}

There are two diagrams to compute, shown in Fig. 1. Using the Feynman rules of the previous subsection for vertices and propagators, one gets

$$
\begin{aligned}
\Pi^{\mu \nu}(P)= & \frac{\widetilde{e}^{2}}{2} \sum_{e= \pm} \int \frac{d^{4} K}{(2 \pi)^{4}} \operatorname{Tr}\left[\gamma^{\mu} \widetilde{Q} \mathcal{S}^{e}(K) \gamma^{\nu} \widetilde{Q} \mathcal{S}^{e}(K-P)\right] \\
& -\frac{\widetilde{e}^{2}}{2} \sum_{e= \pm} \int \frac{d^{4} K}{(2 \pi)^{4}} \operatorname{Tr}\left[\gamma^{\mu} \widetilde{Q} \Xi^{e}(K) \gamma\right. \\
& \left.\times \nu \widetilde{Q} \Xi^{-e}(K-P)\right],
\end{aligned}
$$

where the trace above is in color, flavor and spinor spaces. After the explicit evaluation of the color and flavor traces, one finds

$$
\begin{aligned}
\Pi^{\mu \nu}(P)= & \frac{\widetilde{e}^{2}}{2} \sum_{e= \pm} \int \frac{d^{4} K}{(2 \pi)^{4}}\left\{\operatorname{Tr}\left[\gamma^{\mu} S^{e}(K) \gamma^{\nu} S^{e}(K-P)\right]\right. \\
& \left.+\operatorname{Tr}\left[\gamma^{\mu} \Xi^{e}(K) \gamma^{\nu} \Xi^{-e}(K-P)\right]\right\} \\
& +\frac{\widetilde{e}^{2}}{2} \sum_{e= \pm} \int \frac{d^{4} K}{(2 \pi)^{4}} \operatorname{Tr}\left[\gamma^{\mu} S_{0}^{e}(K) \gamma^{\nu} S_{0}^{e}(K-P)\right] .
\end{aligned}
$$

We find two types of contribution to the photon self-energy. The first one is due to the quarks that form Cooper pairs. This contribution is totally analogous to the one found in the CFL phase, Eq. (2.27), with, however, two main differences. First, the angles $\theta_{\mathrm{CFL}} \neq \theta_{2 \mathrm{SC}}$ are unequal, and therefore $\widetilde{e}$ differs in the CFL and 2SC cases. Second, the numerical factors in front of the integrals are different, which is due to the different charge assignments of the four charged quarks in the CFL and the 2SC phases (integer in the first case, half-integer in the second, cf. Tables II and V). Hence, in the $2 \mathrm{SC}$ phase $c=1$. The second contribution is due to the charged gapless up quark. It is totally analogous to the contribution to the photon self-energy of a charged fermion in a dense medium with chemical potential $\mu$, whose value is well known.

We will not give explicit results for evaluating the spinor traces and the sum over Matsubara frequencies. These can be inferred from Eqs. (2.28), after the necessary changes in numerical factors for the part of the polarization tensor due to the condensed quarks, as mentioned above, and as well for the gapless quark, where furthermore one has to put $\Delta=\bar{\Delta}$ $=0$.

\section{Photon self-energy at low momentum}

We perform the analytical continuation to Minkowski space-time, and study the photon polarization tensor for different values of the external momentum and frequency. We restrict the study to the zero temperature case.

Let us decompose the polarization tensor into longitudinal and transverse parts as done in Eq. (2.30). Here, and in contrast to the CFL phase, the transverse and longitudinal components consist of two different pieces,

$$
\begin{aligned}
& \Pi_{L}\left(p_{0}, \mathbf{p}\right)=\Pi_{L}^{\mathrm{gap}}\left(p_{0}, \mathbf{p}\right)+\Pi_{L}^{\mathrm{HDL}}\left(p_{0}, \mathbf{p}\right), \\
& \Pi_{T}\left(p_{0}, \mathbf{p}\right)=\Pi_{T}^{\mathrm{gap}}\left(p_{0}, \mathbf{p}\right)+\Pi_{T}^{\mathrm{HDL}}\left(p_{0}, \mathbf{p}\right) .
\end{aligned}
$$

The contributions $\Pi^{\text {gap }}$ stem from the condensates and correspond to the first sum in Eq. (3.10). More explicitly, $\Pi^{\text {gap }}$ is given by Eq. (2.28) with $c=1$ in the $2 \mathrm{SC}$ phase. In turn, the contribution $\Pi^{\mathrm{HDL}}$ stems from the free charge carriers, the gapless quark and the electrons. The quark contribution corresponds to the second sum in Eq. (3.10). The corresponding electron contribution reduces also to a hard dense loop. The longitudinal and transverse components of the hard dense loop polarization tensor are given by

$$
\begin{aligned}
\Pi_{L}^{\mathrm{HDL}}\left(p_{0}, \mathbf{p}\right)= & \tilde{m}^{2} f t\left[\frac{p_{0}}{2 p}\left(\ln \left|\frac{p_{0}+p}{p_{0}-p}\right|-i \pi \Theta\left(p^{2}-p_{0}^{2}\right)\right)-1\right], \\
\Pi_{T}^{\mathrm{HDL}}\left(p_{0}, \mathbf{p}\right)= & -\tilde{m}^{2} \frac{p_{0}^{2}}{2 p^{2}}\left[1+\frac{1}{2}\left(\frac{p}{p_{0}}-\frac{p_{0}}{p}\right)\right. \\
& \left.\times\left(\ln \left|\frac{p_{0}+p}{p_{0}-p}\right|-i \pi \Theta\left(p^{2}-p_{0}^{2}\right)\right)\right] .
\end{aligned}
$$


The Debye mass due to the gapless up quarks and the electrons is

$$
\tilde{m}^{2}=\frac{\widetilde{e}^{2} \mu^{2}}{\pi^{2}}+\frac{\widetilde{e}^{2} \mu_{e}^{2}}{\pi^{2}}
$$

where we have supposed that the mass of the electron can be neglected $\left(m_{e} \ll \mu_{e}\right)$.

Let us first consider the deep infrared limit where both $p_{0}, p \ll \Delta$. The part of the self-energy Eq. (3.10) due to the gapless up quark and the electron is given by Eq. (3.12). The part due to the quarks forming Cooper pairs is similar to the CFL case Eq. (2.32). To quadratic order in $p_{0}, p$ the terms $\Pi^{\text {gap }}$ in Eq. (3.11) become

$$
\begin{aligned}
& \Pi_{L}^{\mathrm{gap}}\left(p_{0}, p\right)=-\tilde{\kappa} p^{2}, \\
& \Pi_{T}^{\mathrm{gap}}\left(p_{0}, p\right)=-\tilde{\kappa} p_{0}^{2} .
\end{aligned}
$$

Here, $\tilde{\kappa}$ is given by Eq. (2.33), except that the numerical factor $c=1$ in the $2 \mathrm{SC}$ phase. The dielectric constant $\tilde{\epsilon}$ in the 2SC medium is defined as

$$
\tilde{\epsilon}=1+\tilde{\kappa}=1+\frac{1}{18 \pi^{2}} \frac{\tilde{e}^{2} \mu^{2}}{\Delta^{2}}
$$

Notice that the dielectric constants in the 2SC and CFL phases, Eq. (3.15) and Eq. (2.34) respectively, are different. This comes about because, first, the mixing angles $\theta_{\mathrm{CFL}}$ $\neq \theta_{2 \text { SC }}$ and thus the effective charges are different, and second, because the charge assignments for the four gapped quarks, and hence the numerical prefactors, are different in the two phases.

While the HDL contribution is responsible for Debye screening,

$$
\lim _{p \rightarrow 0} \Pi_{L}(0, \mathbf{p})=-\tilde{m}^{2}
$$

the polarization effects due to the condensate do not create a Meissner mass associated with the photon,

$$
\Pi^{0 i}(0, \mathbf{p})=\Pi^{i j}(0, \mathbf{p})=0 .
$$

For small photon momentum and frequency, Eqs. (3.11) tell us that there are two different types of screening phenomenon: one associated with the existence of condensed quarks, responsible for strong polarization effects, and one associated to the existence of free charge carriers, responsible for Debye screening. It is however straightforward to see that Debye screening is dominant in the infrared limit.

Considering the region where $p_{0}, p \gg \Delta$, but $p_{0}, p \ll \mu$, the contribution to the photon polarization tensor due to the condensed quarks reduces to a HDL, to leading order. The full polarization tensor reduces to Eq. (3.12), with the Debye mass $\tilde{m}^{2}$ replaced by

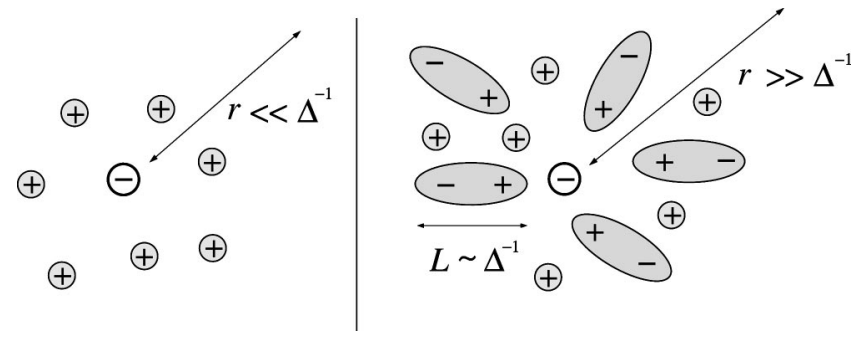

FIG. 3. Screening of a negative test charge in the 2SC phase. Left panel: for length scales $r$ with $(\widetilde{e} \mu)^{-1} \sim r \ll \Delta^{-1}$, the screening is dominated by the ungapped and the effectively ungapped charged quarks. Here, these are the up quarks of colors 1, 2 and 3, leading to the Debye mass $\tilde{M}$. Right panel: For length scales $r \gg \Delta^{-1}$, all up quarks of color 1 or 2 are bound with down quarks of color 2 or 1 to $\widetilde{Q}$-neutral condensates, while the up quark of color 3 remains gapless. The condensates have an electrical dipole moment and modify the dielectric constant. The gapless up quarks still screen the test charge.

$$
\widetilde{M}^{2}=\frac{2 \widetilde{e}^{2} \mu^{2}}{\pi^{2}}+\frac{\widetilde{e}^{2} \mu_{e}^{2}}{\pi^{2}}
$$

In this momentum regime, all charged (gapped or ungapped) quarks contribute to the Debye mass.

\section{E. Discussion}

We briefly discuss the main physical picture that has emerged from our analysis for the 2SC phase. Under the rotated $U(1)$ symmetry, quarks have obtained integer and half-integer electric charges. All half integer charged up and down quarks form electrically neutral Cooper pairs. The gapless up quark has integer charge. The condensates have an electric dipole moment, but no magnetic dipole, because their total angular momentum and spin vanish. Therefore, photon propagation in a $2 \mathrm{SC}$ medium is affected by both the free charged particles and the condensate.

The photon propagation is not affected by the condensate if the photon wavelength is smaller than the size of the Cooper pairs $\sim \Delta^{-1}$. In this regime, the photon resolves the constituents of the bound state and scatters with the charge carriers. Consequently, the photon self-energy equals its counterpart of the non-superconducting phase, with photons being Landau damped, the electric fields acquiring a Debye mass but without a static screening of magnetic fields. The electric Debye mass $\widetilde{M}$ is of the order $\sim \widetilde{e} \mu$ and stems from all charged quarks in the $2 \mathrm{SC}$ phase.

The photon can no longer resolve the diquark condensate for photon wavelengths large compared to the size of the Cooper pairs. Therefore, it does not scatter from the constituents. However, as in the CFL phase, the Cooper pairs still modify the photon propagation by inducing a large dielectric constant to the medium. The magnetic permeability of the medium is not changed. In addition, the ungapped charged quarks still lead to Debye screening in the long wavelength limit.

In the static limit, the resulting picture is as follows (see Fig. 3): We introduce a negative test charge to the supercon- 
ductor which provides a static potential. At distances $r$ $\sim(\widetilde{e} \mu)^{-1} \ll \Delta^{-1}$, the electric test charge is screened by effectively free charge carriers, in the present case up quarks of colors 1,2 and 3 . The associated static potential is given by $V(r)=\widetilde{e} \exp (-\tilde{M} r) /(4 \pi r)$. At larger distances, $r \gg \Delta^{-1}$, Debye screening is less efficient because some of the quarks are bound into Cooper pairs, leading to a strong electric susceptibility $\tilde{\epsilon} \gg 1$. The gapless quark (and the electron) still lead to Debye screening.

\section{SUMMARY AND OUTLOOK}

In this paper we have studied the propagation of electromagnetic fields in the presence of diquark condensates at weak coupling and high baryonic density. The condensates break spontaneously both the non-Abelian gauge symmetry and the $U(1)$ symmetry of electromagnetism, leaving a remaining "rotated" $U(1)$ symmetry unbroken. The gauge field associated with this new symmetry, a linear combination of the real photon and a gluon, plays the role of the in-medium photon in the superconductor. The phenomemon is analogous to what occurs in the electroweak sector of the Standard Model, where the Higgs condensate breaks both the $S U(2)_{L}$ and $U(1)_{Y}$ symmetries, but leaves the $U(1)_{\text {e.m. }}$ unbroken.

We have computed the in-medium photon polarization tensor to one-loop order in the quark fields. In both the CFL and the 2SC phases, the diquark condensate is responsible for a large increase of the dielectric constant of the medium, because the Cooper pairs act as strong electrical dipoles. This effect makes the photon propagation in color superconducting media different from the propagation in vacuum or in a dense medium within the normal phase.

We also found that the magnetic permeability of the medium remains as in the vacuum theory, because the primary condensates have no magnetic moment. Although condensation in the $J=0$ channel is favored, secondary condensates with non-vanishing angular momentum may form in the 2SC or CFL phase $[6,8]$. In those cases, we expect that a nonvanishing magnetic moment of the condensates modifies the magnetic permeability of high density QCD.

An important qualitative difference between $2 \mathrm{SC}$ and CFL matter is due to the fact that all quarks condense and acquire a gap in the CFL phase, while in the 2SC phase, some of the charged quarks do not form primary condensates and remain gapless. In the absence of electrons in CFL matter [30], this implies for the electromagnetic interactions that electric charges are not screened. In turn, the remaining free charge carriers of $2 \mathrm{SC}$ matter always provide a screening for electric charges.

Here, we restricted the discussion to the case of vanishing temperature. It will be interesting to study photon propagation in high density QCD at low temperatures, and, more generally, the related transport properties of the medium. Most transport coefficients like thermal or electrical conductivities, flavor diffusion or shear viscosities are dominated by light degrees of freedom. A first step toward a transport theory for color superconducting QCD has been made in [15], where a transport equation for the gapped quarks of
2SC matter has been discussed. We leave a more detailed study of these questions for future investigations.

\section{ACKNOWLEDGMENTS}

C.M. thanks S. Heinemeyer, E. Lopez and R. Pisarski for useful discussions. This work was supported by the European Community through HPMF-CT-1999-00404 and HPMF-CT-1999-00391.

\section{APPENDIX: PHOTON SELF-ENERGY AND CHARGED SCALAR MESONS}

Here, we compute the contributions to the photon polarization tensor due to charged scalar mesons in the CFL phase. For the discussion in the main text, we are interested in its finite part. We show that contributions from charged scalar mesons can be neglected, compared to the quark contribution, in the infrared limit. We restrict the discussion to the case of vanishing temperature. The main computation is fully equivalent to the computation of the one-loop polarization tensor for scalar QED, which we recall as well (see, for example, Refs. [37,38]).

\section{One-loop polarization tensor of scalar QED}

Consider electrically charged scalar fields with charge $\widetilde{e}$ and mass $m$. To one-loop order, two diagrams with charged scalar fields propagating within the loops contribute to the photon polarization tensor. In Euclidean space-time, it can be written as [38]

$$
\begin{aligned}
\Pi_{\mu \nu}^{\mathrm{qed}}\left(P, m^{2}\right)= & \widetilde{e}^{2} \int \frac{d^{d} K}{(2 \pi)^{d}} \\
& \times\left[\frac{-2 \delta_{\mu \nu}}{K^{2}+m^{2}}+\frac{(2 P+K)_{\mu}(2 P+K)_{\nu}}{\left[(P+K)^{2}+m^{2}\right]\left(K^{2}+m^{2}\right)}\right] .
\end{aligned}
$$

Here, $P^{\mu} \equiv\left(p_{0}, \mathbf{p}\right)$. The first term in the large square brackets stems from the tadpole diagram. Using the Feynman parametrization for the loop integrals, and performing a partial integration, we end up with the following expression:

$$
\Pi_{\mu \nu}\left(P, m^{2}\right)=\left(P_{\mu} P_{\nu}-P^{2} \delta_{\mu \nu}\right) \hat{\Pi}\left(P^{2}, m^{2}\right),
$$

where

$$
\begin{aligned}
\hat{\Pi}\left(P^{2}, m^{2}\right)= & \frac{\widetilde{e}^{2}}{(4 \pi)^{d / 2}} \Gamma\left(2-\frac{d}{2}\right) \int_{0}^{1} d x(1-2 x)^{2} \\
& \times\left[x(1-x) P^{2}+m^{2}\right]^{d / 2-2} .
\end{aligned}
$$

The difference between scalar and spinor QED amounts to the replacement $(1-2 x)^{2} \rightarrow 8 x(1-x)$ in the integrand of Eq. (A3) [38]. Notice that the dimensions of $\hat{\Pi}$ and $\Pi_{\mu \nu}$ are different. From Eq. (A3), and after analytical continuation to Minkowski space, we deduce that the polarization tensor has a cut and acquires an imaginary part for $P^{2} \geqslant 4 m^{2}$ in $d \leqslant 4$ 
dimensions. Within dimensional regularization, and after expanding in $\epsilon=(4-d) / 2$, we find both the divergent and finite parts of the one-loop self-energy:

$$
\begin{aligned}
\hat{\Pi}\left(P^{2}, m^{2}\right)= & \frac{\widetilde{e}^{2}}{(4 \pi)^{2}}\left[\frac{1}{3 \epsilon}+\frac{1}{3}(\gamma-\ln 4 \pi)+\frac{1}{3} \ln \frac{m^{2}}{\Lambda^{2}}-\frac{8}{9}\right. \\
& -\frac{8}{3} \frac{m^{2}}{P^{2}}+\frac{2}{3}\left(1+4 \frac{m^{2}}{P^{2}}\right)^{3 / 2} \\
& \left.\times \operatorname{arccoth} \sqrt{1+4 \frac{m^{2}}{P^{2}}}+\mathcal{O}(\epsilon)\right] .
\end{aligned}
$$

Here, $\gamma$ is the Euler constant, and $\Lambda$ denotes an arbitrary renormalization scale. Let us denote the renormalized part of Eq. (A3) as $\hat{\Pi}_{R}\left(P^{2}, m^{2}\right)=\hat{\Pi}\left(P^{2}, m^{2}\right)-\hat{\Pi}\left(P=0, m^{2}\right)$.

We are interested in computing the finite part of the oneloop self-energy in the infrared limit. For $P^{2} / m^{2} \ll 1$, Eq. (A3) can be Taylor expanded, and we find

$$
\hat{\Pi}_{R}\left(P^{2}, m^{2}\right)=\frac{\widetilde{e}^{2}}{(4 \pi)^{2}}\left[\frac{1}{30} \frac{P^{2}}{m^{2}}-\frac{1}{420} \frac{P^{4}}{m^{4}}+\ldots\right] .
$$

In the static limit where $P^{2}=-p^{2}$, the one-loop correction to the photon self-energy modifies the dielectric constant of vacuum as

$$
\epsilon=1-\frac{\tilde{\alpha}}{120 \pi} \frac{p^{2}}{m^{2}},
$$

where $\tilde{\alpha}=\widetilde{e}^{2} /(4 \pi)$. This result was first noticed by Uehling in the context of spinor QED [38]. The physical interpretation of this result is the following: quantum fluctuations create virtual pairs of positively and negatively charged particles, which act as electric dipoles and contribute to the dielectric constant of the vacuum. The quantum vacuum is not really empty, but full of virtual pairs of particles which are continually created and destroyed.

From Eq. (A3) one can consider as well the opposite limit $-P^{2} / m^{2} \gg 1$. The finite part of the renormalized Green function then reduces to

$$
\hat{\Pi}_{R}\left(P^{2}, m^{2}\right)=\frac{\tilde{\alpha}}{12 \pi}\left[-\ln \frac{\left|P^{2}\right|}{m^{2}}+\frac{8}{3}+\ldots\right] .
$$

\section{One-loop polarization tensor of mesons in the CFL phase}

Now we turn to the computation of the photon polarization tensor due to the charged pions and kaons in the CFL phase. We denote the meson masses as $m_{\pi^{ \pm}}$and $m_{K^{ \pm}}$. They are not yet known, as the contribution arising from the electromagnetic interactions has not been computed. Nevertheless, we know that their masses have to be $m_{s} \ll 2 \Delta$ (with $s$ $=\pi^{ \pm}, K^{ \pm}$), as otherwise they would not be stable. A rough estimate given in Ref. [24] and dimensional analysis suggest that $m_{s} \sim \widetilde{e} \Delta$ in the chiral limit. Furthermore, at vanishing baryonic density, the mesons travel at the speed of light. In contrast, within CFL superconducting matter, their velocity is $v_{\pi}<1$, as can be read off from Eq. (2.12).

We can infer the one-loop contribution to the photon selfenergy in the CFL phase from the vacuum result of scalar QED, by rescaling both the spatial derivatives and the spatial gauge fields as $\partial_{\mu} \rightarrow \widetilde{\partial}_{\mu} \equiv\left(\partial_{0}, v_{\pi} \partial_{i}\right) \quad$ and $\quad A_{\mu} \rightarrow \widetilde{A}_{\mu}$ $\equiv\left(A_{0}, v_{\pi} A_{i}\right)$. Using the Feynman rules as implied by Eq. (2.12), we find that for a charged Goldstone boson field with mass $m_{s}$

$$
\begin{aligned}
\widetilde{\Pi}_{\mu \nu}(P)= & \frac{\widetilde{e}^{2}}{\left(v_{\pi}\right)^{d-1}} \int \frac{d^{d} \widetilde{K}}{(2 \pi)^{d}}\left[\frac{-2 \delta_{\mu \nu}}{\widetilde{K}^{2}+m_{s}^{2}}\right. \\
& \left.+\frac{(2 \widetilde{P}+\widetilde{K})_{\mu}(2 \widetilde{P}+\widetilde{K})_{\nu}}{\left[(\widetilde{P}+\widetilde{K})^{2}+m_{s}^{2}\right]\left(\widetilde{K}^{2}+m_{s}^{2}\right)}\right]
\end{aligned}
$$

with Euclidean momenta $\widetilde{P}^{\mu}=\left(p_{0}, v_{\pi} \mathbf{p}\right)$. The explicit evaluation of Eq. (A8) is simplified by noticing that Eq. (A8) reduces to Eq. (A1), the polarization tensor for scalar QED in vacuum:

$$
\widetilde{\Pi}_{\mu \nu}\left(p_{0}, \mathbf{p}\right)=\left(v_{\pi}\right)^{-3} \Pi_{\mu \nu}^{\mathrm{qed}}\left(p_{0}, v_{\pi} \mathbf{p}\right) .
$$

Finally, we undo the rescaling and equate $A_{\mu} \Pi_{\mu \nu} A_{\nu}$ $\equiv \widetilde{A}_{\mu} \widetilde{\Pi}_{\mu \nu} \widetilde{A}_{\nu}$, in order to find the following components of $\Pi_{\mu \nu}$ :

$$
\begin{aligned}
\Pi^{00}(P) & =\frac{p^{2}}{v_{\pi}} \hat{\Pi}_{R}\left(\widetilde{P}^{2}, m_{s}^{2}\right), \\
\Pi^{0 i}(P)= & -\frac{p^{0} p^{i}}{v_{\pi}} \hat{\Pi}_{R}\left(\widetilde{P}^{2}, m_{s}^{2}\right), \\
\Pi^{i j}(P)= & {\left[\left(\frac{p_{0}^{2}}{v_{\pi}}+v_{\pi} p^{2}\right) \delta^{i j}-v_{\pi} p^{i} p^{j}\right] } \\
& \times \hat{\Pi}_{R}\left(\widetilde{P}^{2}, m_{s}^{2}\right) .
\end{aligned}
$$

This polarization tensor obeys the Ward identity $P_{\mu} \Pi^{\mu \nu}=0$. Decomposing Eqs. (A10)-(A12) as in Eq. (2.30), we find

$$
\begin{aligned}
& \Pi_{L}(P)=\frac{p^{2}}{v_{\pi}} \hat{\Pi}_{R}\left(\widetilde{P}^{2}, m_{s}^{2}\right) \\
& \Pi_{T}(P)=\left(\frac{p_{0}^{2}}{v_{\pi}}+v_{\pi} p^{2}\right) \hat{\Pi}_{R}\left(\widetilde{P}^{2}, m_{s}^{2}\right),
\end{aligned}
$$

the longitudinal and transverse parts of the mesonic contribution to the photon self-energy.

\section{Discussion}

The infrared limit of this polarization tensor can be obtained from Eq. (A5). At this stage, it is easy to see why the low momentum contribution of charged pions and kaons in the CFL phase is negliglible as compared to the quark contribution as computed in Sec. II E. Consider the longitudinal 
polarization tensor in the static limit. From Eq. (A13), we deduce that the leading contribution from the charged mesons for $p \ll m_{s}$ is proportional to $\left(\widetilde{e}^{2} p^{4} v_{\pi}\right) / m^{2}$. For $p \ll \Delta$, the quarks contribute proportionally to $\left(\widetilde{e}^{2} p^{2} \mu^{2}\right) / \Delta^{2}$. Comparing the two expressions, it is obvious that the meson contribution is always negligible, because $\mu / \Delta \gg 1$, while $p / m_{s}$ $\ll 1$. Therefore, as pointed out in the main text, the photon polarization tensor is fully dominated by the quark loops in the infrared limit.

Let us now discuss the intermediate regime where $\Delta$ $\ll p_{0}, p \ll \mu$. We consider the longitudinal polarization tensor in the static limit. As has been shown in Eqs. (2.35) and (2.36), the quark contribution leads to a Debye mass $\Pi_{L}^{\text {quark }}$ $=\left(4 \widetilde{e}^{2} \mu^{2}\right) / \pi^{2}$. From Eqs. (A7) and (A13), we deduce that the meson contribution is given by

$$
\Pi_{L}^{\text {meson }}=\frac{\widetilde{e}^{2} p^{2}}{24 \pi^{2} v_{\pi}}\left[-\ln \frac{p^{2} v_{\pi}^{2}}{m^{2}}+\frac{8}{3}\right] .
$$

Here, we assumed that $p \gg m_{s} / v_{\pi}$. While the explicit values of the meson masses are unknown, their scale is set by the gap $m_{s} \propto \Delta$. In this case, we can perform a leading logarithmic approximation in the non-Abelian gauge coupling. For sufficiently small $g$, the logarithm is dominated by the exponential suppression of the gap [6]. To leading logarithmic accuracy, and in the momentum regime considered, we have $\ln \left[\left(p^{2} v_{\pi}^{2}\right) /\left(m^{2}\right)\right]=\left(6 \pi^{2}\right) /(\sqrt{2} g)+\mathcal{O}(\ln g)+\mathcal{O}(1) . \quad$ Consequently, $\left|\Pi_{L}^{\text {meson }}\right|=\left(\widetilde{e}^{2} p^{2}\right) /\left(4 \sqrt{2} v_{\pi} g\right)$ to leading order in $g$. Although the contribution itself is enhanced by $1 / g$, it remains subleading compared to the quark contribution for $p^{2} \ll\left(16 \sqrt{2} v_{\pi} g \mu^{2}\right) / \pi^{2}$. Dropping irrelevant numerical factors, the bound reads $p \ll \sqrt{g} \mu$. Notice that the $\widetilde{e}$ has disappeared from this estimate, because the leading logarithmic approximation is entirely due to $g \rightarrow 0$. We emphasize that this estimate is a worst-case analysis. For larger $g$, the logarithm is less dominant and the boundary where meson and quark contributions are comparable is pushed toward higher momenta.
[1] B.C. Barrois, Nucl. Phys. B129, 390 (1977).

[2] D. Bailin and A. Love, Phys. Rep. 107, 325 (1984).

[3] M. Alford, K. Rajagopal, and F. Wilczek, Phys. Lett. B 422, 247 (1998); R. Rapp, T. Schäfer, E.V. Shuryak, and M. Velkovsky, Phys. Rev. Lett. 81, 53 (1998).

[4] M. Alford, K. Rajagopal, and F. Wilczek, Nucl. Phys. B537, 443 (1999).

[5] K. Rajagopal and F. Wilczek, hep-ph/0011333.

[6] D.T. Son, Phys. Rev. D 59, 094019 (1999).

[7] T. Schäfer and F. Wilczek, Phys. Rev. D 60, 114033 (1999).

[8] R.D. Pisarski and D.H. Rischke, Phys. Rev. D 61, 051501 (2000); 61, 074017 (2000).

[9] D.K. Hong, Phys. Lett. B 473, 118 (2000); D.K. Hong, V.A. Miransky, I.A. Shovkovy, and L.C. Wijewardhana, Phys. Rev. D 61, 056001 (2000).

[10] W.E. Brown, J.T. Liu, and H. Ren, Phys. Rev. D 61, 114012 (2000); 62, 054016 (2000).

[11] C. Manuel, Phys. Rev. D 62, 114008 (2000).

[12] K. Rajagopal and E. Shuster, Phys. Rev. D 62, 085007 (2000).

[13] D.H. Rischke, Phys. Rev. D 62, 034007 (2000).

[14] D.H. Rischke, D.T. Son, and M.A. Stephanov, Phys. Rev. Lett. 87, 062001 (2001)

[15] D.F. Litim and C. Manuel, Phys. Rev. Lett. 87, 052002 (2001).

[16] R. Casalbuoni and R. Gatto, Phys. Lett. B 464, 111 (1999).

[17] D.T. Son and M.A. Stephanov, Phys. Rev. D 61, 074012 (2000); 62, 059902(E) (2000).

[18] M. Rho, A. Wirzba, and I. Zahed, Phys. Lett. B 473, 126 (2000).

[19] D.K. Hong, T. Lee, and D. Min, Phys. Lett. B 477, 137 (2000).
[20] C. Manuel and M.H. Tytgat, Phys. Lett. B 479, 190 (2000).

[21] K. Zarembo, Phys. Rev. D 62, 054003 (2000).

[22] S.R. Beane, P.F. Bedaque, and M.J. Savage, Phys. Lett. B 483, $131(2000)$

[23] V.A. Miransky, I.A. Shovkovy, and L.C. Wijewardhana, Phys. Rev. D 63, 056005 (2001).

[24] C. Manuel and M.H. Tytgat, Phys. Lett. B 501, 200 (2001).

[25] M. Alford, J. Berges, and K. Rajagopal, Nucl. Phys. B571, 269 (2000).

[26] D.K. Hong, Phys. Rev. D 62, 091501 (2000).

[27] R. Casalbuoni, Z. Duan, and F. Sannino, Phys. Rev. D 63, 114020 (2001).

[28] D.H. Rischke, Phys. Rev. D 62, 054017 (2000).

[29] E.V. Gorbar, Phys. Rev. D 62, 014007 (2000).

[30] K. Rajagopal and F. Wilczek, Phys. Rev. Lett. 86, 3492 (2001).

[31] D.H. Rischke, Phys. Rev. D 64, 094003 (2001).

[32] R.D. Pisarski, Phys. Rev. Lett. 63, 1129 (1989); E. Braaten and R.D. Pisarski, Nucl. Phys. B337, 569 (1990).

[33] C. Manuel, Phys. Rev. D 53, 5866 (1996).

[34] D.K. Hong, M. Rho, and I. Zahed, Phys. Lett. B 468, 261 (1999).

[35] M.A. Nowak, M. Rho, A. Wirzba, and I. Zahed, Phys. Lett. B 497, 85 (2001).

[36] R. Casalbuoni, Z. Duan, and F. Sannino, Phys. Rev. D 62, 094004 (2000).

[37] C. Schubert, "Quantum Field Theory Amplitudes in the String-Inspired Formalism," Lecture notes, 1998 (unpublished).

[38] C. Itzykson and J. B. Zuber, Quantum Field Theory (McGrawHill, New York, 1980). 